\title{
Chaperone protein HSC70 regulates intercellular transfer of Y chromosome antigen DBY
}

\author{
Sascha Kretschmann, ${ }^{1}$ Stefanie Herda, ${ }^{2}$ Heiko Bruns, ${ }^{1}$ Josefine Russ, ${ }^{2}$ Edith D. van der Meijden, ${ }^{1,3}$ Ursula Schlötzer-Schrehardt, ${ }^{4}$ \\ Marieke Griffioen, ${ }^{3}$ II-Kang Na, ${ }^{2,5,6,7}$ Andreas Mackensen, ${ }^{1}$ and Anita N. Kremer ${ }^{1}$ \\ 'Department of Internal Medicine 5, Hematology/Oncology, Friedrich Alexander University Erlangen-Nuremberg, Erlangen, Germany. ${ }^{2}$ Experimental and Clinical Research Center, Berlin, Germany. \\ ${ }^{3}$ Department of Hematology, Leiden University Medical Center, Leiden, Netherlands. ${ }^{4}$ Department of Ophthalmology, Friedrich Alexander University Erlangen-Nuremberg, Erlangen, Germany. ${ }^{5}$ Department \\ of Hematology, Oncology and Tumor Immunology, Charité-Universitätsmedizin Berlin, corporate member of Freie Universität Berlin, Humboldt-Universität zu Berlin, and Berlin Institute of Health, Berlin, \\ Germany. ${ }^{6}$ Berlin-Brandenburg Center of Regenerative Therapies, Berlin, Germany. ${ }^{7}$ Berlin Institute of Health, Berlin, Germany.
}

\begin{abstract}
Recent studies have demonstrated that $\mathrm{CD}^{+} \mathrm{T}$ cells can efficiently reject MHC-II-negative tumors. This requires indirect presentation of tumor-associated antigens on surrounding antigen-presenting cells. We hypothesized that intercellular transfer of proteins is not the sole consequence of cell death-mediated protein release, but depends on heat-shock cognate protein 70 (HSC70) and its KFERQ-like binding motif on substrate proteins. Using human Y chromosome antigen DBY, we showed that mutation of one of its 2 putative binding motifs markedly diminished $\mathrm{T}$ cell activation after indirect presentation and reduced protein-protein interaction with HSC70. Intercellular antigen transfer was shown to be independent of cellcell contact, but relied on engulfment within secreted microvesicles. In vivo, alterations of the homologous KFERQ-like motif in murine DBY hampered tumor rejection, $T$ cell activation, and migration into the tumor and substantially impaired survival. Collectively, we show that intercellular antigen transfer of DBY is tightly regulated via binding to HSC70 and that this mechanism influences recognition and rejection of MHC-II-negative tumors in vivo.
\end{abstract}

\section{Introduction}

$\mathrm{CD}^{+} \mathrm{T}$ cells play a central role in orchestrating innate and adaptive immune responses. Upon recognition of their cognate antigen in MHC-II molecules, $\mathrm{CD}^{+} \mathrm{T}$ cells become activated and secrete cytokines, thereby activating macrophages (1), stimulating B cells to produce antibodies (1), providing help to maturation of dendritic cells (2), and inducing and maintaining $\mathrm{CD} 8^{+}$cytotoxic $\mathrm{T}$ lymphocytes (3-5). While this works perfectly for extracellular pathogens, such as bacteria, that are taken up by professional antigen-presenting cells (APCs) and are processed into MHC-II molecules, antigens from intracellular threats, such as viruses or transformed cells, have to be transferred to surrounding APCs to induce a potent immune response. Indeed, several studies have shown that $\mathrm{CD} 4^{+} \mathrm{T}$ cells reject MHC-II-negative tumor cells in vivo (6-8), indicating induction and also execution of a $\mathrm{CD} 4^{+} \mathrm{T}$ cell-initiated immune response without direct $\mathrm{T}$ cell-target cell interaction. To achieve this, indirect activation of $\mathrm{CD} 4^{+} \mathrm{T}$ cell tumor-derived antigens need to be transferred to surrounding APCs for processing and presentation on MHC-II.

Therefore, it remains controversial whether intercellular antigen transfer is a sole consequence of cell death-mediated antigen release or follows controlled mechanisms by the host.

Conflict of interest: The authors have declared that no conflict of interest exists. Copyright: (5) 2019, American Society for Clinical Investigation.

Submitted: June 25, 2018; Accepted: May 2, 2019; Published: June 17, 2019.

Reference information: J Clin Invest. 2019;129(7):2952-2963.

https://doi.org/10.1172/JCl123105.
Indirectly presented antigens are believed to be released through cell debris or after premature cell death by necrosis (9), both of which intensely occur after invasive therapies (10). In contrast, we (11) and others $(12,13)$ have previously shown that certain antigens cannot be indirectly presented, suggesting a more selective mechanism of antigen release. Vital cells can control their antigen release, for example, by sequestration in membrane vesicles of endosomal origin, known as exosomes $(14,15)$. Indeed, we have demonstrated that minor antigen protein tyrosine kinase $2 \beta$ is released within secreted extracellular vesicles, leading to indirect recognition of previously antigen-negative target cells (11). Moreover, we demonstrated that engulfment within secreted microvesicles at least partially depends on protein structures outside of the $\mathrm{T}$ cell epitope. Interestingly, in a murine model of fibrosarcoma, vesicle-bound antigen was demonstrated to induce a more potent immune response when compared with soluble antigen (16). In support of the latter, priming of naive myeloma-specific $\mathrm{CD} 4^{+} \mathrm{T}$ cells was shown to be stronger after secretion of the tumor-specific antigen compared with local injection or to nonsecreting MHC-IInegative tumor cells (6). These investigations indicate that antigen release by vesicular carriers is controlled and has a lasting and high potential to induce immune responses. Hence, it is of high interest to unravel the molecular biology defining intercellular antigen transfer.

Interestingly, another study has demonstrated a selective microautophagy-like pathway in which certain cytosolic proteins can be delivered to the vesicles of late endosomes (LE) (17). This process was shown to be distinct from chaperone- 
A

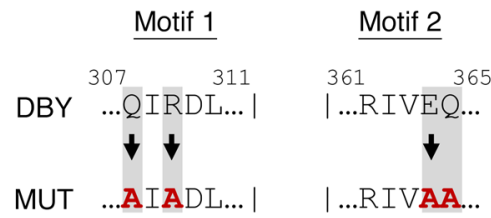

B

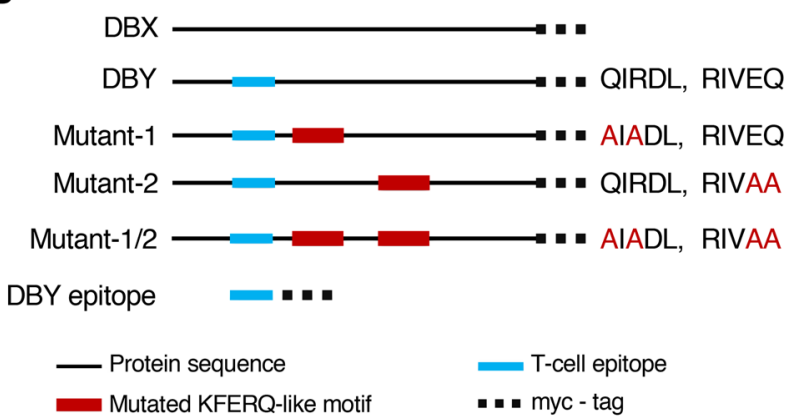

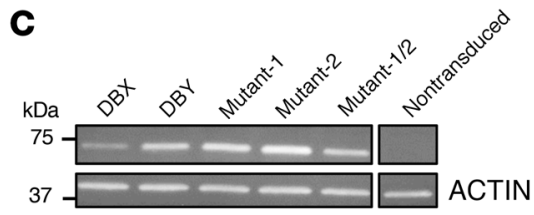

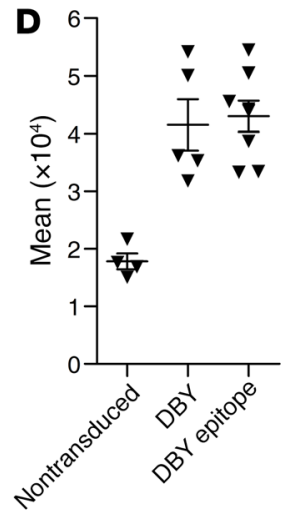

Figure 1. Characterization of putative KFERQ-like motifs in human DBY and retrovirally transduced HeLa cells. (A) Depicted are the 2 identified KFERQ-like motifs in human DBY (motifs 1 and 2), each of which is displayed in its WT (DBY) and mutated representation (MUT). Letters represent amino acids (one letter code) and numbers give information on the protein position. (B) Schematic representation of human DBX and DBY protein constructs illustrating the relative position of mutated KFERQ-like motifs (red bars), the CD4+ $\mathrm{T}$ cell epitope (blue bars), and the fused myc-tag at the $\mathrm{C}$ terminus (dashed line). Representation of WT or mutant KFERQ-like motifs is shown on the right. (C) Western blot analysis of retrovirally transduced HeLa cells. (D) Depicted is the mean fluorescence intensity per cell of retrovirally transduced HeLa cells from a single immunofluorescence imaging experiment. Data are shown as mean \pm SEM and were quantified using ImageJ software (NIH).

mediated autophagy at the lysosomal-limiting membrane (18). In brief, heat-shock cognate protein 70 (HSC70)was reported to bind folded substrate proteins via KFERQ-like motifs (19) to deliver the cargo across the endosomal limiting membrane. It was demonstrated that internalization of substrate proteins to the vesicles relied on the endosomal sorting complexes required for transport (ESCRT) I and III, but did not involve lysosome-associated membrane protein type-2A (LAMP-2A).

Taking into account that proteins delivered to LE can leave the cell when entering the biogenesis of exosomes $(14,15$, 20), the described microautophagy-like process (17) could not only selectively influence recruitment into intraluminal vesicles of the LE, but could also regulate intercellular transfer of certain proteins. Therefore, to investigate involvement of the microautophagy-like process, we analyzed the role of HSC70 as a central regulator in this process in intercellular transfer of cellular antigens and whether this has an impact on $\mathrm{T}$ cell recognition and rejection of MHC-II-negative tumors.

Here, we show that the human Y chromosome antigen DBY can be transferred between cells and that 1 of 2 putative KFERQlike motifs is involved in $\mathrm{CD}^{+} \mathrm{T}$ cell activation upon indirect presentation in vitro. In vivo, the mutation of this KFERQ-like motif in the murine homologue of DBY resulted in premature tumor growth and reduced survival. In conclusion, our data suggest an important role of HSC7O in regulating intercellular antigen transfer,which opens up new perspectives in the field of targeted anticancer strategies.

\section{Results}

Indirect presentation of human $Y$ chromosome antigen $D B Y$ in vitro requires protein structures outside of the $T$ cell epitope. By analyzing the amino acid sequence of human $\mathrm{Y}$ chromosome antigen DBY, we identified 2 pentameric sequences (Figure 1A) following the described characteristics of KFERQ-like motifs $(18,19)$. To analyze the influence of these motifs on intercellular transfer of DBY, we cloned full-length human DBY, fulllength $\mathrm{X}$ chromosome homologue $\mathrm{DBX}$, the $\mathrm{CD}^{+} \mathrm{T}$ cell DBY epitope (amino acids 175-188) (21), and fulllength DBY with mutations in either one or both identified KFERQ-like structures (Figure 1B).

In order to investigate the role of HSC70 in regulating intercellular antigen transfer of human DBY, we retrovirally transduced a human HLA-II-negative cervix carcinoma cell line (HeLa) with the cloned DBY antigen variants. To achieve comparable expression of the transgenes, transduced HeLa cells were single cell sorted based on marker gene expression (Supplemental Figure 1A; supplemental material available online with this article; https://doi. org/10.1172/JCI123105DS1), and transgene expression was confirmed by Western blot analysis (Figure 1C). Due to its low molecular weight (2.9 kDa), the DBY epitope could not be visualized on the immunoblot, but we confirmed expression of this transgene by real-time PCR (Supplemental Figure 1B). Furthermore, to make sure that the transgene was also present at the protein level, we performed immunofluorescence imaging (Supplemental Figure 1C) and additionally quantified the mean fluorescence intensity of DBY epitope-expressing HeLa cells compared with WT DBY and DBY-negative cells (Figure 1D).

To confirm the ability of our constructs to be processed and presented to our human DBY-specific $\mathrm{CD}^{+} \mathrm{T}$ cell clone, we retrovirally transduced a HLA-DQ5-positive, but DBY-negative EBVtransformed lymphoblastoid cell line (EBV-LCL) with the cloned DBY variants. Transduced EBV-LCL were cell sorted and subsequently cocultured with the DBY-specific $\mathrm{CD} 4^{+} \mathrm{T}$ cell clone to measure $\mathrm{T}$ cell activation by IFN- $\gamma$ ELISA. By this, we demonstrated processing and presentation of all constructs in HLA-II-positive EBV-LCL (Supplemental Figure 2A).

To examine indirect presentation, we cocultured transgenepositive and HLA-II-negative HeLa cells (donor cells) with HLADQ5-positive, but DBY-negative EBV-LCL (acceptor cells). Neither the donor cells nor the acceptor cells activated the DBY-specific T cell clone when analyzed separately. However, after acceptor cells were cocultured with donor cells, they activated the $\mathrm{T}$ cell clone as measured by IFN- $\gamma$ ELISA (Figure 2A). After indirect presentation, 
A

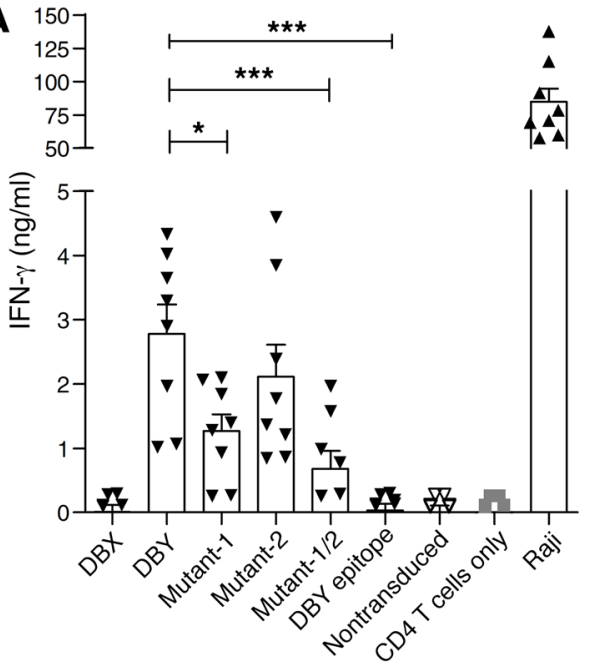

C

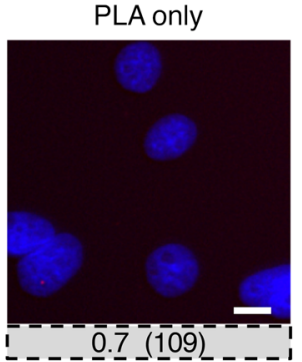

Nontransduced

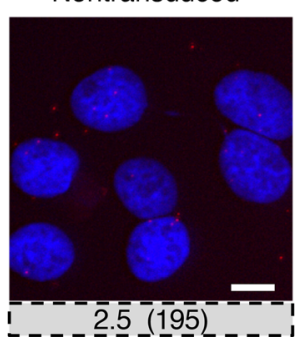

EBV-LCL (HLA $\left.{ }^{\text {pos }} \mathrm{Ag}^{\text {neg }}\right)$

$\boldsymbol{\nabla}+\mathrm{HeLa}\left(\mathrm{HLA}^{\mathrm{neg}} \mathrm{Ag}^{\mathrm{pos}}\right)$

$\nabla+$ HeLa (HLA $\left.{ }^{\text {neg }} A g^{\text {neg }}\right)$

CD4 ${ }^{+} \mathrm{T}$ cells only

$\Delta$ Raji $\left(H L A^{\text {pos }} \mathrm{Ag}^{\text {pos }}\right)$
B

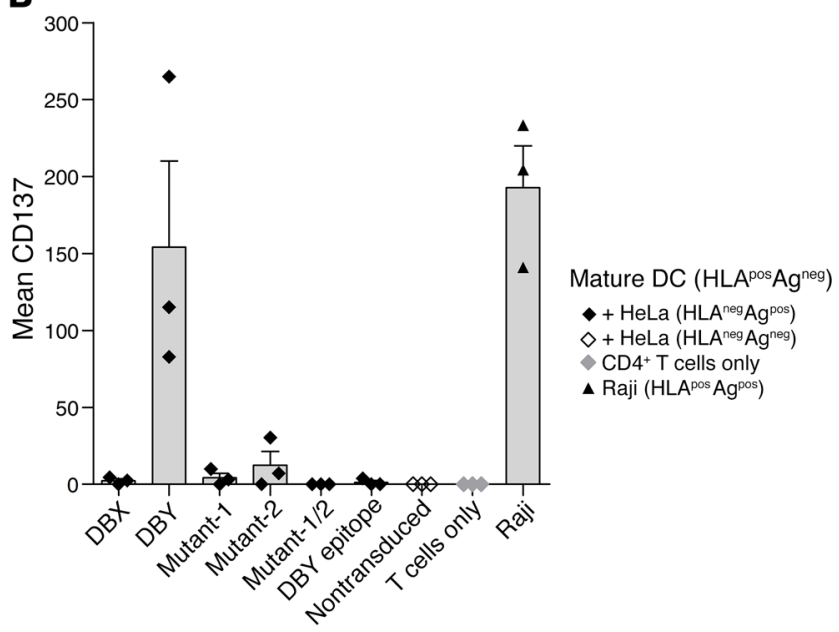

Figure 2. Indirect presentation of full-length human DBY requires protein-protein interaction with HSC70. (A) HLA-II-negative and antigen-positive HeLa cells (HLA ${ }^{\text {neg }} \mathrm{Ag}^{\text {pos }}$ ) were cocultured with HLA-II-positive and antigen-negative EBV-LCL (HLA $\left.{ }^{\text {pos }} A g^{\text {neg }}\right)$. After coincubation, EBV-LCL were isolated and tested for recognition by the DBY-specific CD4+ $\mathrm{T}$ cell clone in IFN- $\gamma$ ELISA. Data are shown as mean \pm SEM of duplicated wells $(n=3-4)$. $P$ values were calculated using 1-way ANOVA with Dunnett's post test. ${ }^{*} P<0.05$; ${ }^{* *} P<0.001$. (B) HLA-II-negative and antigen-positive HeLa cells (HLA ${ }^{\text {neg }}$ Ag ${ }^{\text {pos})}$ were cocultured with mature dendritic cells. After coculture, DBY-specific CD4+ $\mathrm{T}$ cells were added to measure CD137 on T cells after 48 hours by flow cytometry. Data represent mean \pm SEM of single experiments or duplicated wells $(n=2)$. (C) PLA to visualize protein-protein interaction (immunospots) between HSC70 and DBY constructs in HeLa cells. Values correspond to the average quantity of PLA) signals per cell. Values in brackets correspond to the total number of individually analyzed cells from 3-5 different visual fields. DAPI nuclear stain (blue), ligated antibody signal (red). Scale bars: $10 \mu \mathrm{m}$. Original magnification, $\times 400$.

full-length DBY and DBY with mutations in position E364A/Q365A (Mutant-2) induced robust T cell-mediated IFN- $\gamma$ release. Interestingly, $\mathrm{T}$ cell response was significantly reduced for DBY with mutations in position Q307A/R309A (Mutant-1) and for DBY with combined mutations (Mutant-1/2). Moreover, the DBY epitope triggered no $\mathrm{T}$ cell response at all, although it demonstrated activation of the $\mathrm{T}$ cell clone upon direct presentation. We reproduced these results using a human breast adenocarcinoma cell line (MCF7) as a further donor cell line (Supplemental Figure 2, B-D). Beyond that, we replaced our EBV-LCL acceptor cells with monocyte-derived mature dendritic cells (HLA-DQ5-positive female donor) and measured $\mathrm{T}$ cell activation-induced costimulatory molecule CD137 by flow cytometry (Figure 2B). These experiments confirmed reduced indirect presentation and T cell activation of DBY Mutant-1 and Mutant- $1 / 2$ compared with WT DBY. When using mature dendritic cells as acceptor cells, we also observed reduced $\mathrm{T}$ cell activation for DBY Mutant-2, but this did not reach the level of that of DBY Mutant-1 (Figure 2B). These data suggest that intercellular transfer of full-length DBY is regulated by an element outside of the T cell epitope and indicate a crucial role for the putative motif in position 307-311 (QIRDL motif).
Protein-protein interaction between HSC7O and DBY in situ correlates with indirect presentation of $D B Y$ in vitro. Full-length $D B Y$ with alterations in position 307/309 can diminish $\mathrm{T}$ cell activation upon indirect presentation, while the DBY epitope failed to activate the $\mathrm{T}$ cell clone completely. In line with our hypothesis, this would suggest that HSC7O is considerably hampered in binding these particular protein variants. Therefore, we sought to examine close association with HSC70 using an in situ proximity ligation assay (PLA) (22). By this, we showed that HSC70 interacts with fulllength DBY, but not with the short DBY epitope. Of note, protein interaction of HSC70 and full-length DBY Mutant-1 was substantially impaired, as quantified and reflected by the mean of in situ PLA signals per cell (Figure 2C). These findings correlate with our indirect antigen-presentation assay in vitro and further support a role of HSC7O in intercellular transfer of DBY.

Extracellular vesicles of endosomal origin mediate intercellular transfer of DBY. To investigate the nature of antigen transfer, we addressed the question of whether intercellular transfer of DBY is reliant on cell-cell contact. To unravel this issue, supernatants of HeLa cells expressing full-length DBY, full-length DBY Mutant-1, or the DBY epitope were applied to antigen-negative 

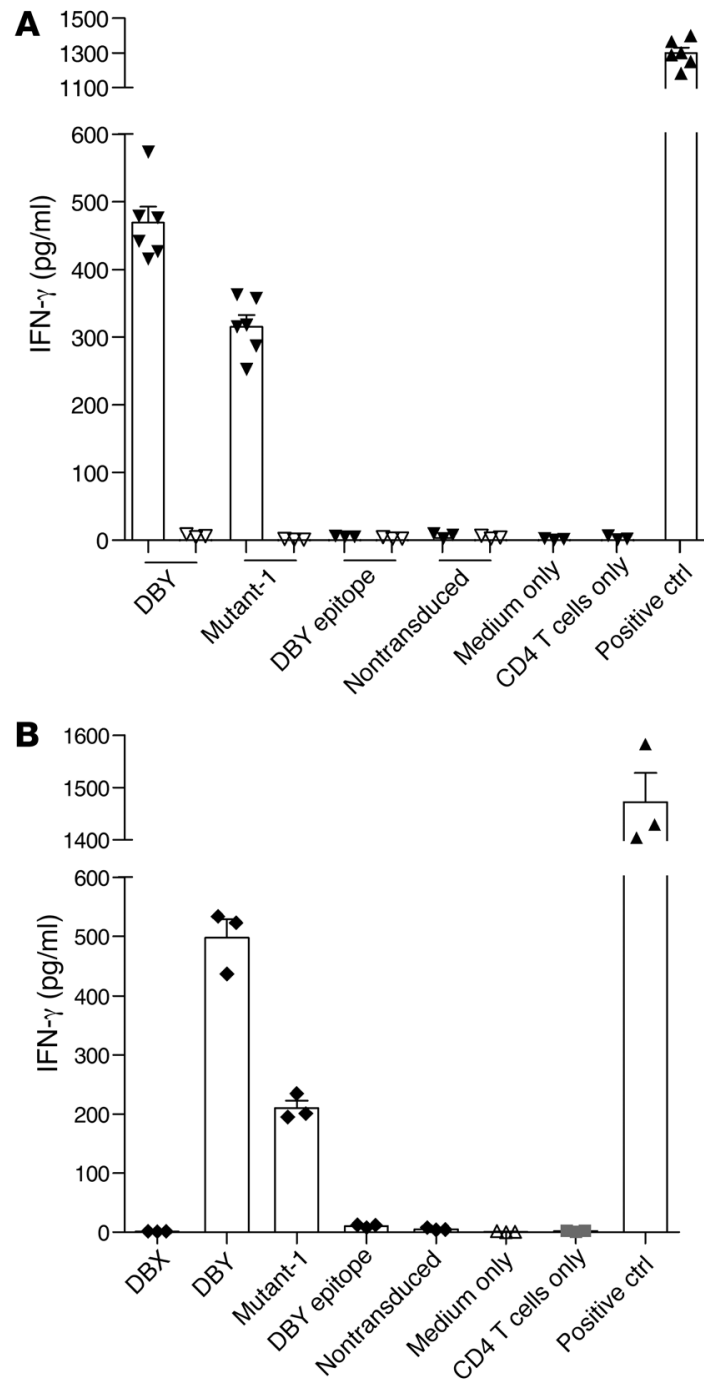

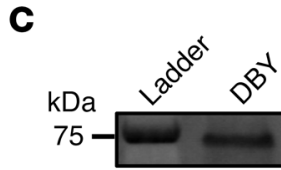

EBV-LCL (HLA $\left.{ }^{\text {pos }} A^{\text {neg }}\right)$

$\checkmark+$ Culture supernatant

$\nabla+$ Filtered supernatant

$\triangle$ EBV-LCL (HLA $\left.{ }^{\text {pos }} \mathrm{Ag}^{\text {pos }}\right)$
EBV-LCL (HLA $\left.{ }^{\text {pos }} A g^{\text {neg }}\right)$

$\checkmark+$ Pellet after 100,000 g

$\Delta+$ Medium only

$\checkmark \mathrm{CD} 4^{+} \mathrm{T}$ cells only

$\triangle$ EBV-LCL (HLA ${ }^{\text {pos }}$ gg $\left.^{\text {pos }}\right)$
D

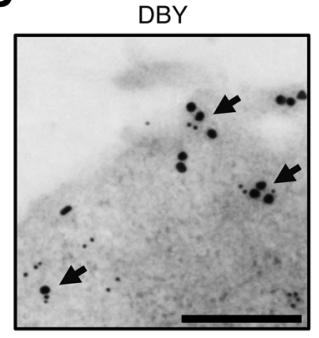

DBY epitope

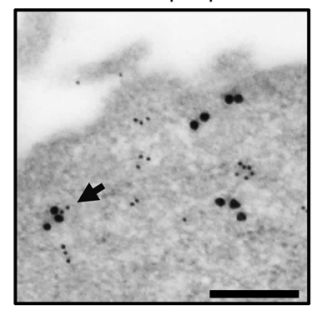

Control

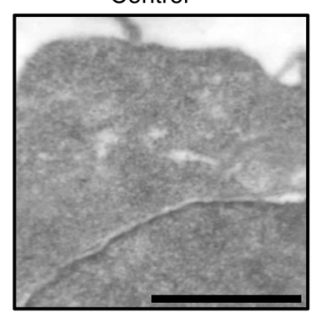

Control

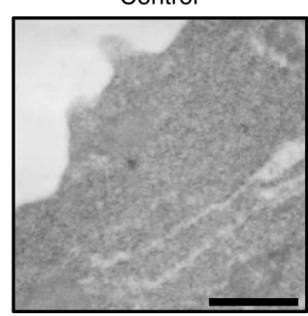

Figure 3. Indirect presentation of full-length human DBY is mediated through extracellular vesicles. (A) Untreated and filtered ( $<100 \mathrm{kDa})$ culture supernatants from serum-free HeLa cell cultures were applied to HLA-II-positive and antigen-negative EBV-LCL (HLA ${ }^{\text {pos }} \mathrm{Ag}^{\text {neg }}$ ) to assess T cell activation in IFN- $\gamma$ ELISA. Data are shown as mean \pm SEM of triplicates $(n=2)$. (B) Culture supernatants of serum-free HeLa cell cultures were ultracentrifuged (100,000 $g$ ), the pellets loaded to HLA-II-positive and antigen-negative EBV-LCL (HLA ${ }^{\text {pos }} A g^{\text {neg }}$ ), and T cell activation measured by IFN- $\gamma$ ELISA. Data represent mean \pm SEM of a representative experiment with triplicate wells. (C) Western blot analysis of the pelleted fraction arising from ultracentrifuged supernatants of HeLa cells expressing full-length DBY. (D) Double immunogold staining on ultrathin sections of HeLa cells expressing full-length DBY and the DBY epitope. Sections were labeled with primary antibody against the transgene-fused myc-tag (small black dots) and CD63 (large black dots). Arrows indicate colocalizations. Scale bars: $0.2 \mu \mathrm{m}$ (DBY); $0.3 \mu \mathrm{m}$ (DBY epitope); $0.5 \mu \mathrm{m}$ (controls).

and HLA-II-positive EBV-LCL and T cell activation was measured by IFN- $\gamma$ ELISA (Figure 3A). We observed $\mathrm{T}$ cell activation for supernatants derived from HeLa cells expressing full-length DBY and the DBY Mutant-1, but not from HeLa cells expressing the DBY epitope. Interestingly, filtration of supernatants (100 $\mathrm{kDa}$ ) abrogated $\mathrm{T}$ cell activation for all antigen variants. These findings suggest that intercellular transfer of our antigens does not require cell-cell contact. Furthermore, the entire absence of $\mathrm{T}$ cell activation after filtration of antigen-positive supernatants suggested that full-length DBY $(74 \mathrm{kDa})$ was recruited to extracellular vesicles. Indeed, protein delivery to LE can result in the formation of intraluminal vesicles destined for secretion as exosomes (15). Therefore, we inspected the role of exosomes in intercellular transfer of DBY and performed serum-free HeLa cell cultures expressing our 3 transgenes of interest. Crude exosomes were purified from culture supernatants by differential ultracentrifugation, and the presence of exosome-associated tetraspanins (CD63, CD81, CD9) (23) was confirmed by flow cytometry (Supplemental Figure 3). Subsequently, the pelleted fractions were loaded to antigen-negative and HLA-II-positive EBV-LCL to measure T cell activation by IFN- $\gamma$ ELISA (Figure 3B). We measured a specific $\mathrm{CD}^{+} \mathrm{T}$ cell activation pattern that was similar to that in our previous coculture studies. Compared with full-length DBY, T cell activation was considerably reduced after loading of exosomes from full-length DBY Mutant-1-expressing cells, while the ultracentrifuged fraction from DBY epitope-expressing cells again triggered no $\mathrm{T}$ cell activation. Beyond this, we performed a Western blot analysis of the loaded fraction from full-length DBY expressing 
A

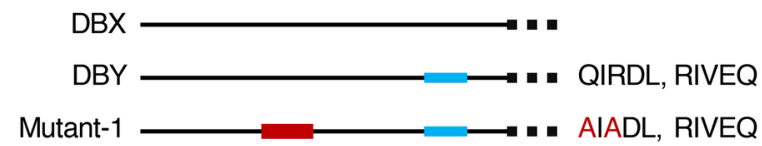

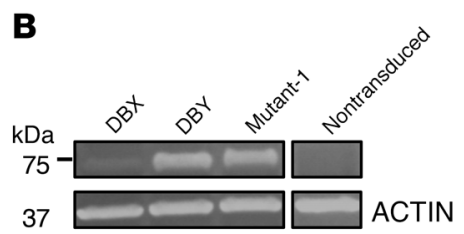
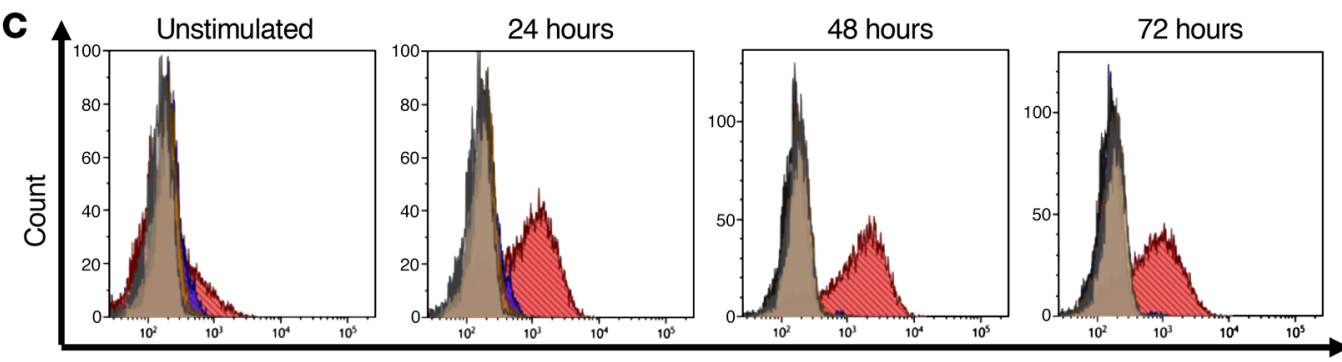

I-Ab

Figure 4. In vitro characterization of retrovirally transduced murine EL-4 cell lines. (A) Schematic representation of murine full-length DBX, fulllength DBY, and DBY Mutant-1 (Q309A/R311A). Representation of WT or mutant KFERQ-like motifs is shown on the right. (B) Western blot analysis of the established EL-4 cell lines. (C) Retrovirally transduced EL-4 cell lines were stimulated with 200 units/ml of murine recombinant IFN- $\gamma$. Surface expression of murine MHC-II (I-Ab) molecules was assessed after 24,48 , and 72 hours by flow cytometry. The murine B cell lymphoma cell line 291 PC was used as a positive control.

cells and detected the full-length antigen inside the pelleted fraction (Figure 3C). The presence of 3 canonical tetraspanins within the loaded fractions suggests that DBY was, indeed, transported to exosomes. However, to independently assess the role of exosomes, we compared electron microscopic imaging of HeLa cells expressing full-length DBY and, in contrast with this antigen, the DBY epitope. Our double-immunogold staining for the exosome surface protein CD63 with either of the transgenes revealed that CD63 and the DBY epitope were colocalized to a clearly lower extent as compared with full-length DBY (Figure 3D).

While HSC7O was reported to bind full-length and folded proteins (17), studies have demonstrated that members of the heat shock protein family 70 (HSP70) can also transport large peptide fragments, which can result in activation of both innate and adaptive immunity (24-26). Therefore, we cloned 2 truncated DBY proteins encoding the T cell epitope and one (DBY 175-314) or both (DBY 175-368) identified KFERQ-like motifs in their WT representation. Despite strong marker gene expression in our cell lines, protein levels were substantially reduced upon immunoblot detection when compared with WT DBY (Supplemental Figure 4, A-D). This result was accompanied by considerably reduced $\mathrm{T}$ cell recognition upon direct antigen presentation and no $\mathrm{T}$ cell recognition upon indirect presentation (Supplemental Figure 5, A and B). Therefore, we cannot exclude contribution of DBY fragments in indirect antigen presentation.

In conclusion, our data suggest that cytosolic DBY is channeled to extracellular vesicles of endosomal origin after binding to HSC70, demonstrating an actively regulated and cell-contactindependent intercellular transfer of this antigen.

Human and murine Y chromosome antigen DBY show conserved KFERQ-like motifs. We demonstrated that alterations in a putative HSC70 binding site in human DBY can affect $\mathrm{T}$ cell activation after indirect presentation in vitro. Next, we addressed the question of the in vivo relevance of $\mathrm{HSC7O}$ in regulating intercellular antigen transfer.
Therefore, we analyzed the amino acid sequence of murine $\mathrm{Y}$ chromosome antigen DBY to identify any putative KFERQ-like motif that meets the criteria (18). Interestingly, we found the same KFERQ-like pentamers, but located at position $+2\left({ }^{(309} \mathrm{QIRDL}^{313}\right.$ and $\left.{ }^{363} \mathrm{RIVEQ}^{367}\right)$ as compared with human DBY (Figure 1A). This indicates conserved putative binding sites for HSC70 between both species.

Alteration in the analog KFERQ-like motif in murine DBY hampers tumor recognition in mice. We used a female murine MHC-IInegative T cell lymphoma cell line (EL-4) and generated tumor cells retrovirally transduced with murine full-length DBX, full-length DBY, and full-length DBY Mutant-1 with alanine substitutions in position Q309A/R311A (Figure 4A). Comparable expression of the transgenes was achieved on the basis of marker gene expression by flow cytometric cell sorting (Supplemental Figure 6A). Additionally, we checked transgene expression in the generated tumor cells at the mRNA (Supplemental Figure 6B) and protein levels (Figure $4 \mathrm{~B})$. While full-length DBY and full-length DBY Mutant-1 were stable and comparably expressed, full-length DBX showed considerably reduced protein stability on the immunoblot. Prior to tumor challenge in a C57BL/6 murine model, we verified the inability of our EL-4 tumor cell lines to express MHC-II molecules (I-Ab). EL-4 cells were stimulated with 200 units of murine recombinant IFN- $\gamma$, and I-Ab expression was analyzed after 24,48 , and 72 hours by flow cytometry (Figure 4C). None of the established cell lines displayed $\mathrm{I}-\mathrm{Ab}$ restriction molecules on the cell surface, which is in line with previous studies reporting that EL-4 shows defective transcription of the MHC-II transactivator (27).

To assess the in vivo relevance, we used the Marilyn mouse model (28) and monitored tumor growth in female mice challenged with the established EL-4 cell lines. Marilyn mice are immunocompromised, but express a transgenic T cell receptor ( $\alpha \mathrm{V}-1.1$ and $\beta \mathrm{V} 6$ ) that recognizes the epitope NAGFNSNRANSSRSS (29) of malerestricted DBY antigen in the context of I-Ab. While tumors 

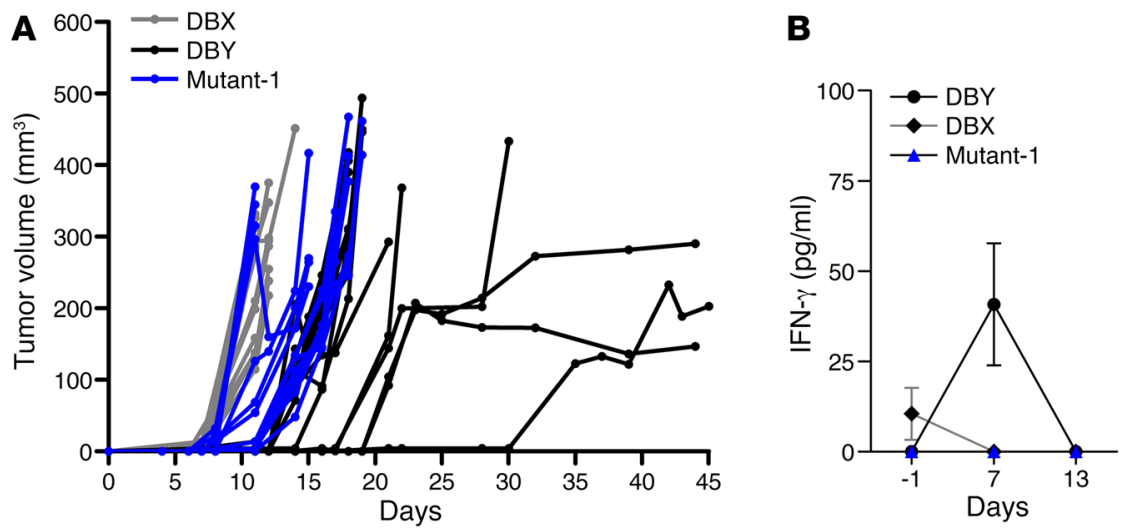

Figure 5. Expression of full-length DBY Mutant-1 in EL-4 tumors significantly reduced survival in Marilyn mice. (A) Tumor growth in female Marilyn mice after challenge with $1 \times 10^{5} \mathrm{EL}-4$ cells expressing antigens as indicated. (B) At indicated days, blood samples were collected and systemic IFN- $\gamma$ was measured by IFN- $\gamma$ ELISA. Data represent mean \pm SEM $(n=5)$. (C) Kaplan-Meier survival curve. The log-rank test was calculated to compare the 2 groups of mice injected with tumors expressing WT DBY (QIRDL) or the DBY Mutant-1 (AIADL). A and C show pooled data from 3 individually performed experiments (DBX, $n=13$; DBY, $n=16$; DBY Mutant-1, $n=15$ ).

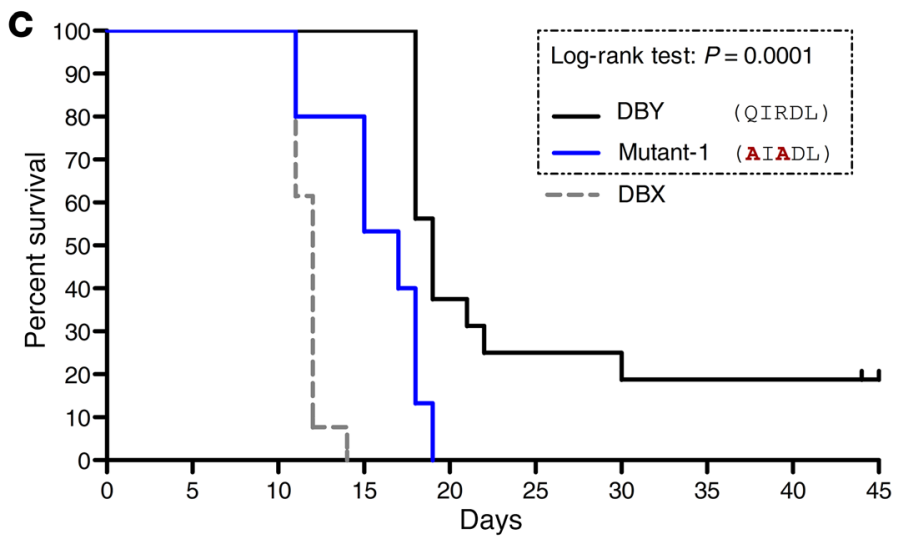

expressing DBX immediately grew out, we observed a delay in outgrowth of tumors expressing WT DBY (Figure 5A). Interestingly, we also observed a slight delay in tumor growth upon challenge with DBY Mutant-1, but this did not reach the level of WT DBY, indicating a somewhat hampered but not completely abolished $\mathrm{T}$ cell response, as previously observed in our in vitro experiments. In line with the proposed $\mathrm{T}$ cell-mediated mechanism of tumor rejection, we detected systemic levels of IFN- $\gamma$ in mice challenged with DBY-expressing tumors, but not upon challenge with DBY Mutant-1- or DBX-expressing tumors (Figure 5B).

However, as DBY-expressing tumors also grew out in the majority of mice, we analyzed expression of the transgene in some tumors and observed a loss of either protein or both protein and mRNA expression in these tumors (Supplemental Figure 7, A-C). In line with the tumor growth data, survival of mice challenged with DBY Mutant-1 tumors was significantly reduced as compared with that of WT DBY (Figure 5C).

In the Marilyn mouse model, $\mathrm{T}$ cell receptor transgenic $\mathrm{T}$ cells most likely encounter their antigen before tumors have established and thus mediate high $\mathrm{T}$ cell pressure on injected tumor cells. To examine indirect antigen presentation in a more clinically relevant setting, we tested our generated EL-4 cell lines in the context of an adoptive T cell therapy (ATT) using a recently published Marilyn mouse model with bioluminescence imaging of T cells (Marilyn-BLITC) on established tumors (30). In addition to survival and tumor growth, this transgenic dual-luciferase reporter system allows longitudinal (migration/expansion) and functional (T cell activation) monitoring of adoptively transferred $\mathrm{T}$ cells via in vivo bioluminescence imaging.
Therefore, albino B6 mice were challenged with EL-4 tumor cells expressing DBX, WT DBY, or the DBY Mutant-1. MarilynBLITC-derived bioluminescence reporter and antigen-specific $\mathrm{CD}^{+} \mathrm{T}$ cells were adoptively transferred by day 12 after tumor challenge. As in the other model, DBX-expressing EL-4 tumors grew out quickly and tumor-bearing mice were all sacrificed by day 6 after ATT. In contrast, tumor size decreased in mice challenged with WT DBY immediately after ATT, whereas mice challenged with tumors expressing the DBY Mutant-1 showed a significant delay in tumor-size reduction (Figure 6A). We measured $\mathrm{T}$ cell migration/expansion and activation by bioluminescence as early as day 4 or day 5 after ATT, respectively (Figure 6, B and C). At that time point, tumor volume was already tremendously decreased for mice bearing WT DBY-expressing tumors, as indicated by the numbers within the bioluminescent images. However, when considering the current tumor volume, we found increased migration/expansion and nuclear factor of activated $\mathrm{T}$ cells-dependent (NFAT-dependent) $\mathrm{T}$ cell activation in mice challenged with WT DBY when compared with mice challenged with the DBY Mutant-1 (Supplemental Figure 8, A and B).

In conclusion, our data illustrate that intercellular transfer of full-length DBY is dependent on HSC70-mediated recruitment through binding to a putative KFERQ-like motif. This recruitment can deliver antigens to extracellular vesicles, which function as antigen carriers between viable cells. Alterations in one putative KFERQ-like motif in DBY diminished T cell activation upon indirect presentation in vitro. This observation was accompanied by marked reduction in tumor rejection and survival upon tumor challenge using 2 distinct murine 
A

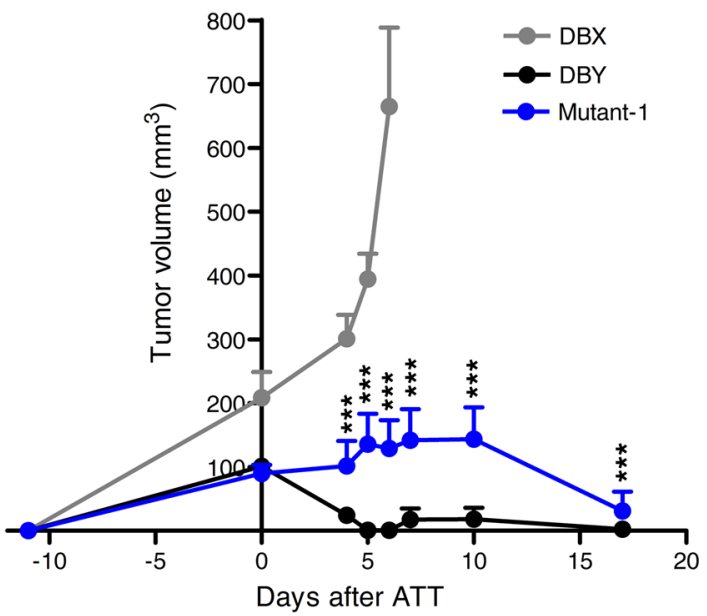

Figure 6. Rejection of established EL-4 tumors expressing full-length DBY Mutant-1 is significantly hampered after ATT. Female albino B6 mice were challenged with $1 \times 10^{5} \mathrm{EL}-4$ tumor cells 12 days prior to the ATT (day 0) with $5 \times 10^{5}$ Marilyn-BLITC CD4+ T cells. (A) Tumor growth in female albino B6 mice after challenge with EL-4 cell lines. At indicated days, $P$ values were calculated by Mann-Whitney $U$ test to compare tumor volumes between DBY and Mutant-1. ${ }^{* *} P<0.001$. Data represent mean \pm SEM ( $n$ $=4-5$ ). (B and C) Recipient mice were analyzed via in vivo bioluminescence imaging for $\mathrm{T}$ cell migration/expansion (Rluc signals in $\mathbf{B}$ ) and T cell activation (NFAT-CBR signals in C). Signal strength is depicted by a pseudocolor scale given in photon $/ \mathrm{s} / \mathrm{cm}^{2} / \mathrm{steradian}\left(\mathrm{p} / \mathrm{s} / \mathrm{cm}^{2} / \mathrm{sr}\right)$. Numbers represent the current tumor volume $\left(\mathrm{mm}^{3}\right)$ measured at indicated days.
B

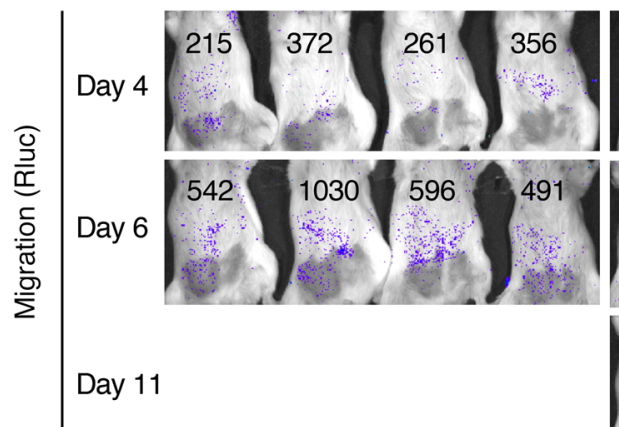

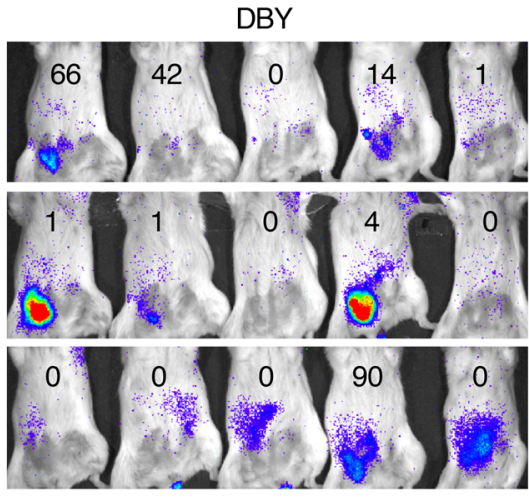
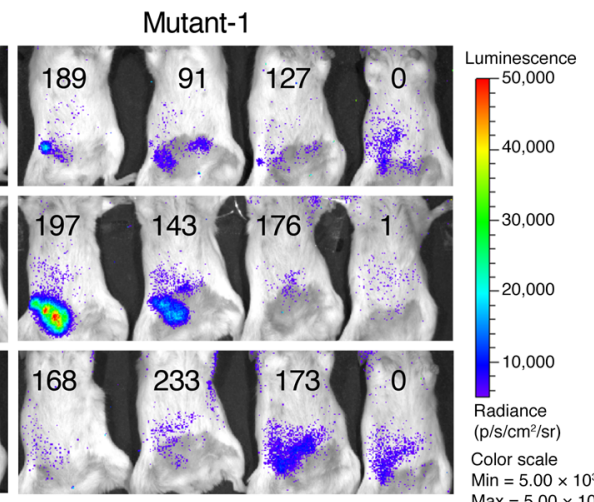

C
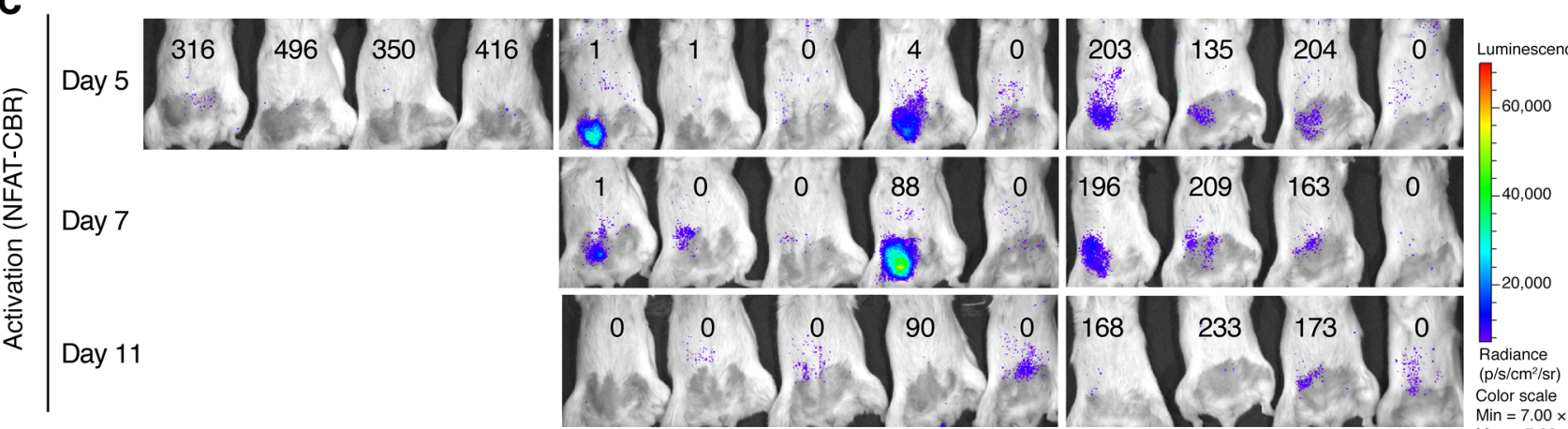

90
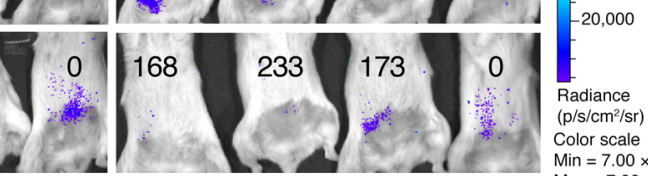

$\left(\mathrm{p} / \mathrm{s} / \mathrm{cm}^{2} / \mathrm{sr}\right)$

Color scale Min $=7.00 \times 10^{3}$
Max $=7.00 \times 10^{4}$

models, indicating a hampered but not completely abolished $\mathrm{T}$ cell response.

These data shed new light on the process of indirect antigen presentation and provide evidence that, beyond autophagy, HSC70 plays an important role in regulating intercellular antigen transfer. This knowledge may help in developing therapeutic strategies for MHC-II-negative tumors and further escape variants.

\section{Discussion}

Immune-escape mechanisms, such as the downregulation of $\mathrm{MHC}$ molecules, can lead to reduced immune recognition, thereby promoting tumor survival (31). Moreover, most solid tumors do not express MHC-II. Nevertheless, several studies reported that CD $4^{+} \mathrm{T}$ cells can efficiently reject MHC-II-negative tumors (6-8). Although it is suggested that tumor antigens are released and presented by surrounding APCs $(8,32-34)$ the underlying mechanisms of T cell activation and tumor rejection in these cases are poorly understood. Here, we showed that human Y chromosome antigen DBY can be transferred from MHC-II-negative tumor cells to surrounding APCs, leading to activation of specific $\mathrm{CD} 4^{+} \mathrm{T}$ cells. This transfer was independent of cell-cell contact, but relied on HSC70-mediated recruitment of full-length proteins into secreted extracellular vesicles. Mutation of a putative HSC70-binding site in DBY not only reduced protein interaction with $\mathrm{HSC70}$, but also diminished $\mathrm{T}$ cell activation in our in vitro presentation assays. In vivo, the same mutation led to substantial reduction in tumor rejection and survival.

HSC70 has been reported to play a central role in chaperonemediated autophagy, where binding of cytosolic proteins to HSC7O facilitates transport via LAMP-2A across the lysosomal-limiting membrane (18). Binding to HSC70 was dependent on so-called 
KFERQ-like motifs within the target proteins. Recently, KFERQlike-mediated binding to HSC70 was also shown to be essential in a process referred to as endosomal microautophagy (17). Thereby, cytosolic proteins are transported into the intraluminal vesicles of the LE by large protein complexes known as the ESCRT machinery (35). Upon fusion of the LE with the lysosome, the content is degraded. About one-third of cytosolic proteins harbor a KFERQ-like motif (36), and it was shown that upon starvation, cytosolic proteins with a KFERQ-like motif are preferentially degraded, reflecting their uptake into lysosomes (19). In contrast to these autophagic pathways, where the cargo is destined for degradation (37), upon fusion of the LE with the plasma membrane, a portion of the intraluminal vesicles are released as exosomes (38), which can in turn transport cellular contents to surrounding cells. It has, however, to our knowledge, never been shown that HSC70 regulates intercellular transfer of cytosolic antigens. Our results show that HSC70-mediated recruitment correlates with intercellular transfer of the antigen and $\mathrm{CD}^{+} \mathrm{T}$ cell activation after indirect presentation. Beside its role in autophagy, this identifies HSC7O as a crucial candidate in regulating intercellular antigen transfer. Therefore, the biological role of intercellular transfer of antigens with a KFERQ-like motif remains unknown, and further studies are required to unravel the crossroad between endolysosomal degradation and extracellular release of KFERQ-like-containing cytosolic proteins.

In the past, it has been demonstrated that members of the heat shock protein family not only bind selectively to peptides and protein fragments (39), but can elicit immune responses and play a role during antigen presentation $(24-26,40)$. Although we could detect full-length DBY in our exosomal fractions, this was based on C-terminal myc-tag staining, and thus, we cannot preclude contribution of C-terminally cleaved fragments. We therefore tried to address the role of large protein fragments in intercellular transfer and T cell activation by generating 2 truncated proteins of human DBY that compose the T cell epitope up to the first or up to the second putative and nonmutated KFERQlike motif. However, these shortened DBY proteins showed reduced protein stability, thereby complicating interpretation of the data. Although we could demonstrate that HSC7O binds to the full-length protein as described for endosomal microautophagy (17), we therefore cannot exclude that peptides of our DBY constructs contribute to intercellular antigen transfer and $\mathrm{T}$ cell activation.

One drawback of our model with retrovirally transduced antigen is the induced overexpression of the target antigen. High cellular expression of proteins has been reported to promote uptake into intraluminal vesicles of the LE via bulk microautophagy (17). However, in our studies, the complete lack of transfer of the sole DBY CD4 ${ }^{+} \mathrm{T}$ cell epitope, which reached comparable or even slightly higher expression levels, argues against this possibility and suggests a strong regulation of this process. Although it might be that the small molecular size leads to differential trafficking, the clear correlation between HSC70 binding and T cell recognition argues for tight $\mathrm{HSC70}$-mediated regulation. Hence, it would be interesting to identify further potential HSC70-binding sites within the target protein and reevaluate antigen transfer for a full-length version of the protein with completely disrupted HSC70 binding. In preliminary data, we generated mutants that indeed further decreased intercellular antigen transfer; however, combining these mutations mostly led to decreased protein stability (data not shown).

As HSC70 functions as chaperone (41), the decreased protein stability of our additional mutants might also indicate successful disruption of HSC70 binding. Another possibility is that different mechanisms collaborate on the recruitment of cytosolic proteins into the intraluminal vesicles of the LE. Interestingly, several posttranslational modifications were found in exosomal proteins that differ from their corresponding cytosolic counterparts (42), suggesting that these modifications might enable uptake into intraluminal vesicles of the LE.

Recently, it was demonstrated that permanent activation of the inhibitory G protein-coupled sphingosine 1-phosphate receptor regulates inward budding on LE and subsequent exosomal maturation (43). Sphingosine 1-phosphate is a cleavage product of ceramides, which were shown to trigger the formation of vesicles in multivesicular endosomes (44). It has also been reported that the ESCRT adapter protein ALIX interacts with syntenin-1 and syndecan to control biogenesis of exosomes (45). We found colocalizations between full-length DBY and exosomal CD63, but whether proteoglycan or ceramide metabolisms intervene in intercellular transfer of this antigen has to be studied in the future. Therefore it might be possible to circumvent compensatory mechanisms of different regulatory pathways.

Finally, we assessed the in vivo role of our findings using 2 distinct murine models of tumor rejection. In the $\mathrm{T}$ cell receptor transgenic Marilyn mouse model, survival was considerably impaired after challenge with tumors expressing the full-length DBY Mutant-1 as compared with those mice challenged with the DBY WT antigen. These data are consistent with our in vitro studies and indicate that intercellular transfer of DBY takes place in vivo and is similarly regulated. Except for 3 mice with stagnated tumor growth, all mice died at a later stage and probably escaped from immune responses. This could be either due to loss of antigen as a result of selection pressure or to overgrowth by contaminating nontransduced tumor cells. Nevertheless, the longer survival as well as the selective detection of systemic IFN- $\gamma$ in mice challenged with WT DBY indicates a selective immune response in these mice, which is at least reduced in mice challenged with mutated DBY. In the future, to strengthen the role of HSC7O binding in in vivo tumor rejection, it would be ideal to have a naturally expressed antigen. This is, however, complicated by the need of mutated variants. In our second mouse model, we monitored tumor rejection via in vivo bioluminescence imaging for $\mathrm{T}$ cell migration and activation using an adoptive transfer of Marilyn-derived antigen-specific $\mathrm{CD} 4^{+} \mathrm{T}$ cells into recipient mice with established EL-4 tumors. In line with the T cell receptor transgenic model, rejection of established EL-4 tumors expressing the DBY Mutant-1 was clearly delayed, whereas those tumors expressing WT DBY were rapidly rejected. Of note, also DBY Mutant-1 tumors are eventually rejected. This is in line with the reduced, but not abolished, presentation of DBY Mutant-1 in our in vitro experiments. It, however, requires further investigation to determine whether the delayed rejection of DBY Mutant-1 tumors can be fully credited on remaining presentation of DBY and recognition by the transferred Marilyn $\mathrm{T}$ cells or whether 
this is based on the endogenous immune system of the recipient Albino B6 mice.

Collectively, in this study, we demonstrate that antigen transfer of human full-length DBY is regulated by binding to HSC70 via the QIRDL motif. Our data further illustrate that HSC70-mediated recruitment promotes delivery of DBY into extracellular vesicles, leading to presentation of tumor-derived antigens on surrounding MHC-II-positive APCs. This mechanism identifies HSC7O as a crucial regulator for intercellular transfer of DBY in vitro and in vivo, as demonstrated by its potential to prolong survival in the $\mathrm{T}$ cell receptor transgenic Marilyn mice as well as to substantially hamper tumor rejection after ATT as shown by bioluminescence imaging on established tumors. To our knowledge, this is the first report showing that HSC70 acts in a microautophagy-like process to regulate intercellular transfer of an MHC-II-restricted antigen.

\section{Methods}

Cell culture. HeLa (ATCC, CCL-2), MCF7 (ATCC, HTB-22), Phoenix-A (ATCC, CRL-3213), Raji (ATCC, CCL-86), EBV-LCL, EL-4 (ATCC, TIB-39), and 291PC (46) were cultured in RPMI 1640 (PAN-Biotech) with $10 \%$ FCS, $40 \mathrm{U} / \mathrm{ml}$ penicillin, $40 \mu \mathrm{g} / \mathrm{ml}$ streptomycin, $2 \mathrm{mM}$ L-glutamine, $0.4 \%$ vitamin solution, $50 \mu \mathrm{M} \beta$-mercaptoethanol, $1 \%$ minimal essential media, and $1 \mathrm{mM}$ sodium pyruvate (all Gibco, Thermo Fisher Scientific). T cells were cultured in RPMI 1640, as mentioned, but with $5 \%$ human serum (PAN-Biotech), 5\% FCS, and 100 IU/ml IL-2 (Proleukin). Every 11 to 14 days, T cells were restimulated with $50 \mathrm{~Gy}$ irradiated allogeneic feeder cells and $0.8 \mu \mathrm{g} / \mathrm{ml}$ phytohemagglutinin (Oxoid), as previously described (47). The human DBYspecific $\mathrm{CD} 4^{+} \mathrm{T}$ cell clone was obtained from a male patient after allogeneic hematopoietic stem cell transplantation with his HLA-identical sister (48) and recognized epitope PHIENFSDIDMGEI (21) in HLADQB1 ${ }^{*}$ 0501. For in vitro stimulation with IFN- $\gamma$, EL-4 cells or 291PC $\left(5 \times 10^{4}\right.$ cells/well $)$ were stimulated with $40 \mu \mathrm{g} / \mathrm{ml}$ of murine recombinant IFN- $\gamma$ (BioLegend). Culture supernatants of cell lines were regularly tested for mycoplasma contamination (Minerva-Biolabs) by PCR.

Flow cytometry and cell sorting. Flow cytometric analyses were performed on a FACSCanto II (BD Biosciences), and cell sorting was carried out on a FACSAria II SORB (BD Biosciences) using PE-labeled mouse monoclonal antibodies against CD271/ANGFR (C40-1457; BD Biosciences - Pharmingen), CD4 (SK3; BD Biosciences - Pharmingen), and CD9 (HI9a; BioLegend). V450-labeled anti-CD63 (H5C6; BD Biosciences - Pharmingen), eFluor 450-conjugated anti-I-Ab (AF6-120.1; Invitrogen), APC-labeled anti-CD81 (JS-81; BD Biosciences - Pharmingen), and anti-CD137 (4B4-1; PD Pharmingen) mouse monoclonal antibodies were used.

Retroviral constructs and transduction. Human and murine fulllength DBX, full-length DBY, full-length DBY mutants, and the human $\mathrm{CD}^{+} \mathrm{T}$ cell epitope PHIENFSDIDMGEI (21) were all fused to a C-terminal myc-tag EQKLISEEDL (49) and cloned into the pMP71 (50) retroviral vector with truncated nerve growth factor receptor $(\triangle \mathrm{NGFR})$ as marker gene. Mutants were generated by a 2-step PCR, and all constructs were sequence analyzed. All oligonucleotides are provided in the Supplemental Materials (Supplemental Tables 1-4). Phoenix-A cells were transfected as previously described (51) with X-tremeGENE HP DNA Transfection Reagent (Roche). EBVLCL, HeLa, and EL-4 cell lines were transduced on culture plates coated with $30 \mu \mathrm{g} / \mathrm{ml}$ recombinant human fibronectin (Takara Shu- zo). Transduction efficiency was measured by flow cytometry with anti-human CD271/ $\triangle$ NGFR antibody.

Antigen-presentation assays. For direct presentation, stimulator cells $\left(3 \times 10^{4}\right.$ cells/well $)$ were cocultured with a human DBY-specific $\mathrm{CD} 4^{+} \mathrm{T}$ cell clone $\left(5 \times 10^{3}\right.$ cells/well) for 18 hours (48). IFN- $\gamma$ release was measured in $100 \mu$ l supernatants by human IFN- $\gamma$ ELISA (eBioscience). For indirect presentation, antigen-positive and HLA-II-negative HeLa cells $\left(3 \times 10^{5}\right.$ cells/well $)$ were first cocultured with antigen-negative and HLA-II-positive EBV-LCL $\left(3 \times 10^{5}\right.$ cells/well $)$ for 3 days. To measure antigen recognition by the DBY-specific $\mathrm{CD}^{+}{ }^{+} \mathrm{T}$ cell clone, EBV-LCL cells were harvested from coculture and treated as described for direct presentation. For indirect presentation with human dendritic cells, monocytes from a female HLA-DQ5-positive donor were first stimulated with human recombinant GM-CSF ( $500 \mathrm{U} / \mathrm{ml}$; Sanofi), IL-4, and TGF- $\beta$ ( $5 \mathrm{ng} / \mathrm{ml}$; PreproTech) for 6 days. Resulting immature dendritic cells ( 1 to $2 \times 10^{4}$ cells/well) were plated on previously seeded HeLa cell lines $\left(3 \times 10^{5}\right.$ cells/well) for 2 days supplemented with GM-CSF (500 $\mathrm{U} / \mathrm{ml}), \mathrm{IL}-4$ (5 ng/ml), IL-1 $\beta$ and TNF- $\alpha$ (10 ng/ml; Promokine), IL-6 (1000 U/ml; CellGenix), and PGE-2 ( $1 \mu \mathrm{g} / \mathrm{ml}$ Enzo Life Sciences) to generate mature dendritic cells. Subsequently, the DBY-specific CD $4^{+}$ $\mathrm{T}$ cell clone was added and CD137 measured after 48 hours by flow cytometry. For application of culture supernatants, antigen-transduced HeLa cells $\left(1.5 \times 10^{5}\right.$ cells/well $)$ were cultured for 3 days. Supernatants were filtered through $100 \mathrm{kDa}$ centrifugal filters (Merck); $100 \mu \mathrm{l}$ of filtered or nonfiltered supernatants were applied to antigen-negative EBV-LCL $\left(3 \times 10^{4}\right.$ cells/well $)$ and the cells incubated for 2 days before DBY-specific CD $4^{+} \mathrm{T}$ cells were added $\left(5 \times 10^{3}\right.$ cells/well $)$ and IFN- $\gamma$ ELISA was performed.

In situ PLA. Antigen-positive HeLa cells $\left(4 \times 10^{5}\right.$ cells/well) were plated into 8-chamber slides (Thermo Scientific). After overnight incubation, cells were washed in $1 \mathrm{ml}$ PBS and fixed for 10 minutes at room temperature in $500 \mu$ lixative (4\% paraformaldehyde in PBS). Cells in chambers were washed 2 more times and treated with $500 \mu$ l permeabilization buffer (0.5\% Triton X-100 in PBS) for 10 minutes at room temperature. Staining and incubations in a humidity chamber were carried out following the PLA probe and detection protocols listed in the user manual of the Duolink In Situ Red Starter Kit (Olink Bioscience). As primary antibodies, monoclonal rabbit anti-human HSPA8 (EP1531Y; OriGene) and monoclonal mouse anti-human myc-tag (9B11; Cell Signaling) were used. Immunofluorescence images were captured using a Biorevo BZ-9000 fluorescence microscope (Keyence). Illustrated photographs show an overlay of the best 4 images along the $z$ axis and were optimized using the black balance tool and haze reduction function provided in the manufacturer's analyzer software. Protein interaction was quantified with the Duolink-ImageTool (Olink Bioscience) and displayed as PLA signal.

Isolation of exosomes. HeLa cells $\left(5 \times 10^{6}\right.$ cells/flask $)$ were stepwise adapted to grow in Ex-Cell-HeLa Serum-Free Medium (SigmaAldrich) and cultured for 4 days. Cells were centrifuged at $500 \mathrm{~g}$ for 5 minutes. Apoptotic bodies and cellular debris were depleted by subsequent centrifugation at $2000 \mathrm{~g}$ for 20 minutes and a further step at $10,000 \mathrm{~g}$ for 1 hour. Obtained supernatants were centrifuged for 2 hours at $100,000 \mathrm{~g}$. Pellets were collected in $200 \mu \mathrm{l} \mathrm{serum-free} \mathrm{medi-}$ um and immediately used in $\mathrm{T}$ cell experiments and for flow cytometric characterization after overnight incubation with anti-CD63 magnetic beads (Invitrogen). For immunoblotting, exosome pellet was collected in $50 \mu \mathrm{l}$ lysis buffer. 
Microscopy. Immunofluorescent analysis of retrovirally transduced HeLa cells was evaluated using an Axiovert 200 fluorescence microscope and the manufacturer's AxioVision LE64 software (Carl Zeiss). HeLa cells $\left(1 \times 10^{3}\right.$ cells/chamber) were grown on 8 -chamber slides and cultured for 3 days. Cells were fixed (4\% paraformaldehyde in PBS) and permeabilized (2\% FCS and $0.3 \%$ Triton X-100 in PBS) before staining with rabbit polyclonal anti-myc tag antibody (ab9106; Abcam), followed by anti-rabbit Alexa Fluor 555 (Cell Signaling) and DAPI nuclear stain (Carl Roth). For postembedding immunogold labeling, HeLa cells $\left(3 \times 10^{6}\right.$ cells/well) were cultured on Thermanox coverslips (Thermo Scientific) for 4 days and fixed in $4 \%$ paraformaldehyde and $0.1 \%$ glutaraldehyde in $0.1 \mathrm{M}$ cacodylate buffer ( $\mathrm{pH}$ 7.4) for 1 hour at $4^{\circ} \mathrm{C}$. Specimens were dehydrated serially to $70 \%$ ethanol at $-20^{\circ} \mathrm{C}$ and embedded in resin (LR White; Electron Microscopy Sciences). Ultrathin sections were successively incubated in TBS, $0.05 \mathrm{M}$ glycine in TBS, $0.5 \%$ ovalbumin, and $0.5 \%$ fish gelatin in TBS, primary antibodies (mouse anti-CD63 and rabbit anti-myc tag) diluted in TBS-ovalbumin overnight at $4^{\circ} \mathrm{C}$, and finally in $10 \mathrm{~nm}$ and $18 \mathrm{~nm}$ gold-conjugated secondary antibodies (BioCell) diluted 1:30 in TBS-ovalbumin for 1 hour. After rinsing, the sections were stained with uranyl acetate and examined with a transmission electron microscope (906E, Carl Zeiss). In negative control samples, the primary antibody was replaced by PBS or equimolar concentrations of nonimmune rabbit IgG or an irrelevant primary antibody.

Quantitative real-time PCR. Total RNA was isolated from donor cells $\left(3 \times 10^{6}\right.$ cells $)$ and complementary DNA synthesized with M-MuLV reverse transcriptase (New England Biolabs GmbH). Quantitative real-time PCR was performed on a StepOnePlus Real-Time PCR system (Applied Biosystems). PCR was performed in $20 \mu \mathrm{l}$ reactions with $100 \mathrm{nM}$ of each primer (Supplemental Table 5) and SYBR select master mix (Applied Biosystems). Expression of target genes was normalized to the housekeeping gene $18 \mathrm{~S}$ ribosomal RNA and calculated using the comparative threshold cycle $(\Delta \Delta \mathrm{CT})$ method (52).

Immunoblotting. Cells $\left(3 \times 10^{6}\right.$ cells/sample $)$ were lysed on ice in RIPA buffer (50 mM Tris, $150 \mathrm{mM} \mathrm{NaCl}, 1 \%$ Triton X-100, $50 \mathrm{mM}$ $\left.\mathrm{NaF}, 0,2 \mathrm{mM} \mathrm{Na}_{3} \mathrm{VO}_{4}, \mathrm{pH} 7,4\right)$ supplemented with protease inhibitors (Roche). Cytosolic proteins were isolated from supernatants after centrifugation at $16,000 \mathrm{~g}$ for 30 minutes. From 10 to $25 \mu \mathrm{g}$ of protein lysate was separated by SDS-PAGE for 1 hour at $120 \mathrm{~V}$ using precast Mini-PROTEAN TGX 4\%-15\% gradient gels (Bio-Rad) and blotted on $0.2 \mu \mathrm{m}$ Trans-Blot Turbo polyvinylidene difluoride membranes (Bio-Rad). Full-length DBX, DBY and DBY mutants were detected by the fused myc-tags with a rabbit polyclonal antibody (ab9106; abcam) and visualized by the WesternDot 625 goat anti-rabbit Western blot kit according to the manufacturer's protocol (Thermo Scientific). For detection of full-length DBY in the crude exosome fraction, the pellet was collected in RIPA buffer and blotted as described above.

Mice. Anti H-Y T cell receptor transgenic Marilyn mice (C57BL/6) were provided by Olivier Lantz (Institute Curie, Paris, France) (28). Marilyn-BLITC mice are T cell receptor transgenic mice expressing Renilla luciferase (Rluc) and the NFAT-dependent click-beetle luciferase (NFAT-CBR); they have been recently described (30). Albino B6 mice (C57BL/6[Cg]-Tyrc-2J) lacking skin and fur pigmentation were used as ATT recipients for increased bioluminescence imaging sensitivity. All mice were kept under specific pathogen-free conditions following institutional guidelines.
Tumor challenge and adoptive transfer. For the tumor challenge, EL-4 cell lines were adjusted to $1 \times 10^{6}$ cells $/ \mathrm{ml}$ in PBS, mixed 1:1 with Matrigel (BD Biosciences), and kept on ice until subcutaneous injection of $100 \mu \mathrm{l}\left(1 \times 10^{5}\right.$ cells $)$ into the right flank of female age-matched mice. For adoptive transfer of T cells, 12 days after tumor challenge, mice received intravenously $5 \times 10^{5} \mathrm{~T}$ cells from Marilyn-BLITC previously primed in vitro and cultured in the presence of IL-15/IL-7 for 7 days (30). Mean tumor volumes were calculated by measuring tumor length and width with a digital caliper (Fine Science Tools) using cylindrical approximation (volume $=$ width $\times$ length $^{2} / 2$ ). Mice were euthanized when tumors exceeded $10 \mathrm{~mm}$ in length or width. Where indicated, blood was collected weekly to determine peripheral blood IFN- $\gamma$ by a mouse IFN- $\gamma$ ELISA set (BD OptEIA).

Bioluminescent imaging. In vivo imaging was performed using a Xenogen IVIS 200 (Caliper Lifescience). In order to analyze Renilla luciferase (Rluc) signals for $\mathrm{T}$ cell migration/expansion, anesthetized mice were imaged 3 minutes after intravenous injection of freshly prepared coelenterazine (Biosynth) dissolved in DMSO (Sigma-Aldrich) and diluted in PBS (100 $\mu \mathrm{g} / \mathrm{mouse})$. To analyze NFAT-dependent click-beetle (NFAT-CBR) signals for T cell activation, mice received intraperitoneal D-luciferin (Biosynth) $(300 \mu \mathrm{g} / \mathrm{g}$ body weight) prepared in PBS and were imaged 10 minutes later. Images were acquired for 5 minutes using small binning. Bioluminescent data were acquired, analyzed, and visualized using Living Image software (PerkinElmer).

Statistics. All graphs were created and statistical analyses performed using Prism 5.03 (GraphPad Software Inc.). One-way ANOVA with Dunnett's post test was used for multiple comparisons. Twotailed Mann-Whitney $U$ test was used to compare 2 groups wherever indicated. For Kaplan-Meier survival curve the log-rank test was performed to compare 2 groups as indicated. For statistical analyses, at least 3 individual experiments were performed and significance indicated. Otherwise, illustrated experiments are representatives of at least 2 independent experiments unless otherwise stated.

Study approval. All animal experiments were conducted at the Max-Delbrück-Center for molecular medicine according to the German Animal Welfare Act and were reviewed and approved (G 0199/17) by the regional authorities (Landesamt für Gesundheit und Soziales Berlin, Germany). Peripheral blood samples were obtained from a healthy person after approval (P12.051) by the Leiden University Medical Center Institutional Review Board and informed consent according to the Declaration of Helsinki.

\section{Author contributions}

SK cloned all genes, established retrovirally transduced cell lines, designed and performed experiments, analyzed data, and wrote the manuscript. SH and JR performed tumor studies in mice under supervision of IKN. HB contributed to immunofluorescence imaging. MG contributed to the design of the project and reviewed the manuscript. EDVDM performed experiments. USS performed electron microscopic imaging. AM contributed to supervision of the project and reviewed the manuscript. ANK designed the project, acquired grants, supervised work, and reviewed the manuscript.

\section{Acknowledgments}

The authors thank the Core Unit Cell Sorting and Immunomonitoring Erlangen for cell sorting and the department of Transfusion 
Medicine and Haemostaseology Erlangen for providing the fluorescence microscope facility. They thank M. Ziegelmann and N. Daberkow-Nitsche for assistance with regulations concerning animal test applications. The authors further thank Olivier Lantz for providing Marilyn mice. This work was supported by the interdisciplinary Center for Clinical Research Erlangen (IZKF, grant J34), the Jung-Foundation, and the SofieWallner-Foundation. Work is also funded by the Deutsche For- schungsgemeinschaft (DFG, German Research Foundation), project numbers 324392634-TRR 221 (A01) and KR 4313/3-1.

Address correspondence to: Anita Natalie Kremer, Department of Internal Medicine 5, Hematology \& Oncology, Friedrich-AlexanderUniversity Erlangen-Nuremberg, Ulmenweg 18, D-91054 Erlangen, Germany. Phone: 49.0.9131.85.43183; Email: anita.kremer@ uk-erlangen.de.
1. Rocha PN, Plumb TJ, Crowley SD, Coffman TM. Effector mechanisms in transplant rejection. Immunol Rev. 2003;196:51-64.

2. Toes RE, Schoenberger SP, van der Voort EI, Offringa R, Melief CJ. CD40-CD40 ligand interactions and their role in cytotoxic $\mathrm{T}$ lymphocyte priming and anti-tumor immunity. Semin Immunol. 1998;10(6):443-448.

3. Janssen EM, Lemmens EE, Wolfe T, Christen U, von Herrath MG, Schoenberger SP. CD4 ${ }^{+}$ $\mathrm{T}$ cells are required for secondary expansion and memory in $\mathrm{CD} 8^{+} \mathrm{T}$ lymphocytes. Nature. 2003;421(6925):852-856.

4. Hung K, Hayashi R, Lafond-Walker A, Lowenstein C, Pardoll D, Levitsky H. The central role of CD4(+) T cells in the antitumor immune response. JExp Med.1998;188(12):2357-2368.

5. Antony $\mathrm{PA}$, et al. $\mathrm{CD} 8^{+} \mathrm{T}$ cell immunity against a tumor/self-antigen is augmented by $\mathrm{CD} 4^{+} \mathrm{T}$ helper cells and hindered by naturally occurring $\mathrm{T}$ regulatory cells. J Immunol. 2005;174(5):2591-2601.

6. Corthay A, et al. Primary antitumor immune response mediated by $\mathrm{CD} 4^{+} \mathrm{T}$ cells. Immunity. 2005;22(3):371-383.

7. Perez-Diez A, et al. CD4 cells can be more efficient at tumor rejection than CD8 cells. Blood. 2007;109(12):5346-5354.

8. Mumberg D, et al. CD4(+) T cells eliminate MHC class II-negative cancer cells in vivo by indirect effects of IFN- $\gamma$. Proc Natl Acad Sci U S A. 1999;96(15):8633-8638.

9. Basu S, Binder RJ, Suto R, Anderson KM, Srivastava PK. Necrotic but not apoptotic cell death releases heat shock proteins, which deliver a partial maturation signal to dendritic cells and activate the NF- $\mathrm{kB}$ pathway. Int Immunol. 2000;12(11):1539-1546.

10. Kaur P, Asea A. Radiation-induced effects and the immune system in cancer. Front Oncol. 2012;2:191.

11. Kremer AN, et al. Natural T-cell ligands that are created by genetic variants can be transferred between cells by extracellular vesicles. Eur J Immunol. 2018;48(10):1621-1631.

12. Philip M, Schietinger A, Schreiber H. Ribosomal versus non-ribosomal cellular antigens: factors determining efficiency of indirect presentation to $\mathrm{CD}^{+} \mathrm{T}$ cells. Immunology. 2010;130(4):494-503.

13. Taylor GS, Long HM, Haigh TA, Larsen M, Brooks J, Rickinson AB. A role for intercellular antigen transfer in the recognition of EBV-transformed B cell lines by EBV nuclear antigen-specific $\mathrm{CD} 4^{+} \mathrm{T}$ cells. J Immunol. 2006;177(6):3746-3756.

14. Wolfers J, et al. Tumor-derived exosomes are a source of shared tumor rejection antigens for
CTL cross-priming. Nat Med. 2001;7(3):297-303.

15. Simons M, Raposo G. Exosomes - vesicular carriers for intercellular communication. Curr Opin Cell Biol. 2009;21(4):575-581.

16. Zeelenberg IS, et al. Targeting tumor antigens to secreted membrane vesicles in vivo induces efficient antitumor immune responses. Cancer Res. 2008;68(4):1228-1235.

17. Sahu R, et al. Microautophagy of cytosolic proteins by late endosomes. Dev Cell. 2011;20(1):131-139.

18. Dice JF. Chaperone-mediated autophagy. Autophagy. 2007;3(4):295-299.

19. Dice JF, Chiang HL. Peptide signals for protein degradation within lysosomes. Biochem Soc Symp. 1989;55:45-55.

20. Bobrie A, Théry C. Exosomes and communication between tumours and the immune system: are all exosomes equal? Biochem Soc Trans. 2013;41(1):263-267.

21. Vogt MH, et al. The DBY gene codes for an HLA-DQ5-restricted human male-specific minor histocompatibility antigen involved in graftversus-host disease. Blood. 2002;99(8):3027-3032

22. Söderberg O, et al. Direct observation of individual endogenous protein complexes in situ by proximity ligation. Nat Methods. 2006;3(12):995-1000.

23. Lin J, et al. Exosomes: novel biomarkers for clinical diagnosis. ScientificWorldJournal. 2015;2015:657086.

24. Udono H, Srivastava PK. Heat shock protein 70-associated peptides elicit specific cancer immunity. J Exp Med.1993;178(4):1391-1396.

25. Srivastava P. Interaction of heat shock proteins with peptides and antigen presenting cells: chaperoning of the innate and adaptive immune responses. Annu Rev Immunol. 2002;20:395-425.

26. Kumaraguru U, Rouse RJ, Nair SK, Bruce BD, Rouse BT. Involvement of an ATP-dependent peptide chaperone in cross-presentation after DNA immunization. J Immunol. 2000;165(2):750-759.

27. Holling TM, Schooten E, van Den Elsen PJ. Function and regulation of MHC class II molecules in T-lymphocytes: of mice and men. Hum Immunol. 2004;65(4):282-290.

28. Lantz O, Grandjean I, Matzinger P, Di Santo JP. Gamma chain required for naïve $\mathrm{CD} 4^{+} \mathrm{T}$ cell survival but not for antigen proliferation. Nat Immunol. 2000;1(1):54-58.

29. Scott D, et al. Dendritic cells permit identification of genes encoding MHC class II-restricted epitopes of transplantation antigens. Immunity. 2000;12(6):711-720.

30. Szyska M, et al. A transgenic dual-luciferase reporter mouse for longitudinal and functional monitoring of T cells. Cancer Immunol Res. 2018;6(1):110-120.

31. Muenst S, Läubli H, Soysal SD, Zippelius A, Tzankov A, Hoeller S. The immune system and cancer evasion strategies: therapeutic concepts. JIntern Med. 2016;279(6):541-562.

32. Corthay A. A three-cell model for activation of naive Thelper cells. Scand J Immunol. 2006;64(2):93-96.

33. Corthay A, Lundin KU, Lorvik KB, Hofgaard PO, Bogen B. Secretion of tumor-specific antigen by myeloma cells is required for cancer immunosurveillance by $\mathrm{CD} 4^{+} \mathrm{T}$ cells. Cancer Res. 2009;69(14):5901-5907.

34. Haabeth OA, et al. How do CD4(+) T cells detect and eliminate tumor cells that either lack or express MHC class II molecules? Front Immunol. 2014;5:174.

35. Raiborg C, Stenmark H. The ESCRT machinery in endosomal sorting of ubiquitylated membrane proteins. Nature. 2009;458(7237):445-452.

36. Chiang HL, Dice JF. Peptide sequences that target proteins for enhanced degradation during serum withdrawal. J Biol Chem. 1988;263(14):6797-6805.

37. Tekirdag K, Cuervo AM. Chaperone-mediated autophagy and endosomal microautophagy: joint by a chaperone. J Biol Chem. 2018;293(15):5414-5424.

38. Bobrie A, Colombo M, Raposo G, Théry C. Exosome secretion: molecular mechanisms and roles in immune responses. Traffic. 2011;12(12):1659-1668.

39. Grossmann ME, et al. Proteomics shows Hsp70 does not bind peptide sequences indiscriminately in vivo. Exp Cell Res. 2004;297(1):108-117.

40. Blachere NE, et al. Heat shock protein-peptide complexes, reconstituted in vitro, elicit peptide-specific cytotoxic T lymphocyte response and tumor immunity. J Exp Med. 1997;186(8):1315-1322.

41. Stricher F, Macri C, Ruff M, Muller S. HSPA8/ HSC7O chaperone protein: structure, function, and chemical targeting. Autophagy. 2013;9(12):1937-1954.

42. Moreno-Gonzalo O, Villarroya-Beltri C, Sánchez-Madrid F. Post-translational modifications of exosomal proteins. Front Immunol. 2014;5:383.

43. Kajimoto T, Okada T, Miya S, Zhang L, Nakamura S. Ongoing activation of sphingosine 1-phosphate receptors mediates maturation of exosomal multivesicular endosomes. Nat Commun. 2013;4:2712.

44. Trajkovic K, et al. Ceramide triggers budding of exosome vesicles into multivesicular endosomes. Science. 2008;319(5867):1244-1247. 
45. Baietti MF, et al. Syndecan-syntenin-ALIX regulates the biogenesis of exosomes. Nat Cell Biol. 2012;14(7):677-685.

46. Gerbitz A, et al. Stromal interferon- $\gamma$ signaling and cross-presentation are required to eliminate antigen-loss variants of B cell lymphomas in mice. PLoS One. 2012;7(3):e34552.

47. Griffioen M, et al. Identification of phosphatidylinositol 4-kinase type II beta as HLA class II-restricted target in graft versus leukemia reactivity. Proc Natl Acad Sci U S A.
2008;105(10):3837-3842.

48. Faber LM, van Luxemburg-Heijs SA, Veenhof WF, Willemze R, Falkenburg JH. Generation of $\mathrm{CD}^{+}$cytotoxic T-lymphocyte clones from a patient with severe graft-versus-host disease after allogeneic bone marrow transplantation: implications for graft-versus-leukemia reactivity. Blood. 1995;86(7):2821-2828.

49. Cravchik A, Matus A. A novel strategy for the immunological tagging of cDNA constructs. Gene. 1993;137(1):139-143.
50. Fehse B, et al. A novel 'sort-suicide' fusion gene vector for $\mathrm{T}$ cell manipulation. Gene Ther. 2002;9(23):1633-1638.

51. Griffioen M, et al. Genetic engineering of virus-specific $\mathrm{T}$ cells with T-cell receptors recognizing minor histocompatibility antigens for clinical application. Haematologica. 2008;93(10):1535-1543.

52. Pfaffl MW. A new mathematical model for relative quantification in real-time RT-PCR. Nucleic Acids Res. 2001;29(9):e45. 